OPEN ACCESS

Edited by:

Gong Cheng,

Tsinghua University, China

Reviewed by:

Fengwei Bai,

University of Southern Mississippi,

United States

Tongling Shan,

Shanghai Veterinary Research

Institute, Chinese Academy of

Agricultural Sciences (CAAS), China

*Correspondence:

Ming Liao

mliao@scau.edu.cn

Wenbao Q

qiwenbao@scau.edu.cn

†These authors have contributed equally to this work

Specialty section:

This article was submitted to Veterinary Infectious Diseases, a section of the journal

Frontiers in Veterinary Science

Received: 30 April 2021

Accepted: 07 July 2021

Published: 02 August 2021

Citation:

Xing J, Zhang Y, Lin Z, Liu L, Xu Q, Liang J, Yuan Z, Huang C, Liao $M$ and

Qi W (2021) 3'UTR SL-IV and DB1 Regions Contribute to Japanese Encephalitis Virus Replication and Pathogenicity.

Front. Vet. Sci. 8:703147. doi: 10.3389/fvets.2021.703147

\section{3'UTR SL-IV and DB1 Regions Contribute to Japanese Encephalitis Virus Replication and Pathogenicity}

\author{
Jinchao Xing ${ }^{1,2,3 \dagger}$, Youyue Zhang ${ }^{1,2,3 \dagger}$, Ziying Lin ${ }^{1,2}$, Lele Liu ${ }^{1,2}$, Qiang $X u^{1,2}$, Jiaqi Liang ${ }^{1,2}$, \\ Zhaoxia Yuan ${ }^{4}$, Cuiqin Huang ${ }^{5}$, Ming Liao ${ }^{1,2,3,6 *}$ and Wenbao Qi ${ }^{1,2,3,6 *}$ \\ ${ }^{1}$ Key Laboratory of Zoonoses, Ministry of Agriculture and Rural Affairs, South China Agricultural University, Guangzhou, \\ China, ${ }^{2}$ National and Regional Joint Engineering Laboratory for Medicament of Zoonoses Prevention and Control, \\ Guangzhou, China, ${ }^{3}$ Guangdong Laboratory for Lingnan Modern Agriculture, Guangzhou, China, ${ }^{4}$ College of Animal \\ Sciences and Technology, Zhongkai University of Agriculture and Engineering, Guangzhou, China, ${ }^{5}$ The Key Laboratory of \\ Fujian Animal Diseases Control, Longyan University, Longyan, China, ${ }^{6}$ Key Laboratory of Zoonoses Prevention and Control \\ of Guangdong Province, Guangzhou, China
}

Japanese encephalitis virus (JEV), a mosquito-borne flavivirus that causes fatal neurological disease in humans, is one of the most important emerging pathogens of public health significance. JEV is maintained in an enzootic cycle and causes reproductive failure in pigs. Notably, the shift in JEV genotypes is not fully protected by existing vaccines, so the development of a candidate vaccine is urgently needed. In this study, we compared pathogenicity between Japanese encephalitis virus SA14 and BJB (isolated from humans in the 1970s) strains. We found that the BJB strain was attenuated in mice and that there was no case fatality rate. The growth rate of BJB was higher than SA14 virus in BHK-21 cells. Based on the sequence alignment of the viral genome between the SA14 and BJB virus strains, some mutations at sites 248, 254, 258, and 307 were observed in the $3^{\prime}$ untranslated region ( $3^{\prime} U T R$ ). The $3^{\prime} U T R$ of JEV plays a very important role in the viral life cycle. Furthermore, using a reverse genetic system, we conducted and rescued the parental JEV strain SA14 (T248, A254, and A258) and the mutant virus rSA14-3'UTRmut (T248C, A254G, A258G, and 307G). Through an analysis of the RNA secondary structure model of the $3^{\prime} U T R$, we discovered that the mutations of T248C, A254G, and A258G reduced the apiculus ring and increased the lateral ring significantly in the stem-loop structures IV (SL-IV) structure region of $3^{\prime} U T R$. Moreover, the insertion of $307 \mathrm{G}$ added a ring to the dumbbell structure 1 (DB1) structure region. Strikingly, these RNA secondary structure changes in 3'UTR of rSA14-3'UTRmut increased viral negative chain RNA production and enhanced the replication ability of the virus in BHK-21 cells. However, in vivo mouse experiments illustrated that the rSA14-3'UTRmut virus significantly decreased the neurovirulence of JEV. These results affirmed that the JEV SL-IV and DB1 regions play an important role in viral proliferation and pathogenicity. Taken together, we complement the study of RNA element function in the $3^{\prime} U T R$ region of JEV by providing a new target for the rational design of live attenuated candidate vaccines and the increase of virus production.

Keywords: Japanese encephalitis virus, $3^{\prime}$ untranslated region, stem-loop IV, virus replication, pathogenicity 


\section{INTRODUCTION}

Japanese encephalitis virus (JEV) belongs to the genus Flavivirus in the family Flaviviridae. Flaviviruses such as dengue virus (DENV), Zika virus (ZIKV), and West Nile virus (WNV) can cause viral encephalitis, Guillain-Barré syndrome, and infant microcephaly (1). JEV is maintained in an enzootic cycle involving mosquito vectors (particularly Culex tritaeniorhynchus) and amplified in the main vertebrate of host pigs and wading birds $(2,3)$. The JEV exists as five distinguishable genotypes (G-I, G-II, G-III, G-IV, and G-V) based on nucleotide homology in the E protein gene $(4,5)$. JEV G-III was the historically dominant genotype throughout most of Asia, but it has been gradually replaced over the last 20 years by G-I in many Asian countries $(6,7)$. However, the shift in JEV genotypes is not fully protected by existing vaccines $(8,9)$. The need for new JE vaccine strategies is urgent.

The JEV genome is a single-stranded, positive-sense RNA about $11 \mathrm{~kb}$ in length, and it encodes a single polyprotein that is processed post-translationally into structural and non-structural proteins by cellular and viral proteases (10). The structural proteins make up the virion (11), and the non-structural proteins have multiple functions during the virus life cycle, including virus replication and host immune evasion (12-14). Significantly, highly conserved secondary structures are formed by the $5^{\prime}$ and $3^{\prime}$ untranslated regions (UTR) and are implicated in virus replication, translation and packaging of the genome (15).

Recently, studies on flaviviruses have shown that the cis-RNA structural elements of the untranslated region of the flavivirus play an important role in immune escape and virus replication in host cells (16-18). For the synthesis of the genomic RNA to take place, the replicase complex must specifically recognize viral cis-acting RNA elements, defined by primary sequences or secondary/tertiary structures (19). These RNA elements are found in various locations within the genome but most frequently are located in the $5^{\prime}$ and $3^{\prime}$ UTRs (17). The hairpin structure of $3^{\prime}$ UTR is specific to the host for DENV, and the point mutation in the hairpin structure eliminated infection by the virus in mosquito cells but did not affect the replication of the virus in mammalian cells $(20,21)$. In vertebrate and invertebrate cells infected with DENV, the restriction of host cells results in selective genetic mutation of the virus, and the virus $3^{\prime} \mathrm{UTR}$ often has a hot spot for mutation $(22,23)$. The $3^{\prime} U T R$ includes four stem-loop structures (SL, named SLI, SLII, SLIII, and SLIV) and two dumbbell structures (DB, named DB1 and DB2). SL and DB structures are essential in the initial virus replication process. SL-II structures are the main components that prevent $5^{\prime}-3^{\prime}$ RNA Xrn1 from degrading and that produce subgenomic flavivirus RNA (sfRNA) (24). This sfRNA plays an important role in causing cell lesions and pathogenicity by participating in the apoptosis of host cells and regulating the host's antiviral type I interferon response (25-27). The growth efficiency of DENV which deleted DB1 or DB2 was reduced (28). In addition, while deletion of DB1 reduced replication of DENV, viruses lacking DB2 displayed a great increase of fitness in mosquitoes (29). Although research on flavivirus UTR has been carried out in depth, the current studies mainly focus on the RNA elements
SL-II and DB2 in the ${ }^{\prime}$ UTR. Research on the RNA element SL-IV and DB1 structures of JEV is still rare.

In this study, we found that the BJB strain, isolated from human in the 1970s, was attenuated in mice, and that there was no case fatality rate. Based on the sequence alignment of the viral genome, we found some mutations at sites 248,254 , and 258 , as well as an insert at site 307 in the $3^{\prime}$ UTR. Through analysis of the RNA secondary structure model of the $3^{\prime} \mathrm{UTR}$, it was found that the nucleotide mutations of rSA14-3' UTRmut caused the top loop to shrink and the side loop to increase in the SL-IV structure region, and a ring was added to the DB1 structure region. These RNA secondary structure changes enhanced the replication of the virus in BHK-21 cells and significantly reduced pathogenicity in mice as well as the ability of neuron invasion. Collectively, this study provides a new perspective for the weakening of candidate vaccine strains and the increase of virus production.

\section{MATERIALS AND METHODS}

\section{Cells and Viruses}

Baby hamster kidney cells (BHK-21) were maintained in Dulbecco's modified Eagle's medium (DMEM; Gibco), supplemented with $5 \%$ fetal bovine serum (FBS; Biological Industries, Israel) at $37^{\circ} \mathrm{C}$ in $5 \% \mathrm{CO}_{2}$. The Japanese encephalitis virus (JEV) strains SA14 (pACYC-SA14, U14163) and BJB were kindly provided separately by Dr. Bo Zhang (Wuhan Institute of Virology, Chinese Academy of Sciences, China) and Dr. Changwen Ke (Guangdong Center for Disease Control and Prevention).

\section{Plasmid Construction}

An infectious cDNA clone of JEV (pACYC-SA14) was used as the parental JEV strain for mutations. The mutant fragment was synthesized by the GENEWIZ company. The infectious cDNA clones pACYC-rSA14-3'UTRmut were handled using XbaI and XhoI sites to clone on the vector, pACYC. The following primers were used $\left(5^{\prime}-3^{\prime}\right): 3^{\prime}$ UTR -F:GGGTCATCTAGTG TGATTTAAGGTAGAAAA， $3^{\prime}$ UTR -R: CCGACCCAGATCT TGTGTTCTTCCTCACCA, HDVr-F: ACTAGGCACAGAG CGCCGAAGTA, XhoI-R: ATTCAACGGGAAACGTCTTGCT CGA, XbaI-F: CATTTGGTTCATGTGGCTTGGAGCACGGTA, NS5-R: CTTAAATCACACTAGATGACCCTGTCTTCC.

\section{RNA Transcription in vitro and Transfection}

The plasmids for the infectious clone were linearized using XhoI, followed in purification by phenol-chloroform extraction. RNA was then transcribed from the linearized plasmids using a T7 mMESSAGE mMACHINE kit (Ambion) according to the manufacturer's instructions. BHK-21 cells in 12- well plates were transfected with vitro-transcribed RNA using the Lipofectamine 2000 transfection reagent (Invitrogen) according to the manufacturer's instructions.

\section{Virus Replication in BHK-21 Cells}

BHK-21 cells were infected with viruses at a multiplicity of infection (MOI) of 0.01 for $1 \mathrm{~h}$ and washed twice with phosphatebuffered saline (PBS) (Gibco), followed by the addition of 
medium. At 24, 48, and $72 \mathrm{~h}$ post-infection (hpi), the medium was harvested, and virus titer was determined in the BHK21 cells.

\section{Virus Titer by TCID $_{50}$ Method}

Confluent monolayers of BHK-21 cells on 12-well plates were infected with wild-type and recombinant JEV and harvested at different time points post-infection. BHK-21 cells on 96-well plates were infected with 10 -fold serial dilutions of the viral stock. 2 days post-inoculation, the infection situation of cells was examined by immunocytochemistry. BHK-21 cells on 96well plates infected with JEV were washed three times with PBS and fixed with pre-cold methanol for $30 \mathrm{~min}$ at $-20^{\circ} \mathrm{C}$. Then, the cells were washed three times with PBS, incubated with primary antibody NS1 (ascitic fluid secreting mouse monoclonal anti-JEV NS1 by hybridoma cells) for $1.5 \mathrm{~h}$ at room temperature (RT), and followed by the appropriate secondary antibody HRP (Jackson ImmunoResearch, 115-035-003) for $1 \mathrm{~h}$ at RT. Finally, the cells were chemically stained with preparation mixture $(1 \mathrm{~mL} 3-$ Amino-9-ethylcarbazole solution (0.4g 3-Amino-9-ethylcarbazol powder, $100 \mathrm{~mL}$ Dimethylformamide), $15 \mathrm{~mL} 0.1 \mathrm{~mol} \mathrm{NaAc}$, and $0.15 \mathrm{~mL} 3 \% \mathrm{H}_{2} \mathrm{O}_{2}$ ) for $30 \mathrm{~min}$ at RT and the number of infected wells were then counted using a microscope. The $50 \%$ tissue culture infection dose $\left(\mathrm{TCID}_{50}\right)$ of each sample was determined using the Reed and Muench method.

\section{Real-Time Reverse Transcription (RT)-PCR}

Total RNA was extracted from cells and tissues with Total RNA Kit I R6834 (OMEGA). The obtained RNA was subjected by using Moloney murine leukemia virus reverse transcriptase (MMLV) (Takara) for specific reverse transcription of the positive and negative strands, respectively. The JEV positive-chain RNA was reverse transcribed using the JEV-Tag-R primer, and GAPDH-R, was amplified using the tag sequence as the reverse primer and JEV-F as the forward primer. The JEV negative-chain RNA was reverse transcribed synthesis using the JEV-Tag-F primer and GAPDH-R, was amplified using the tag sequence as the forward primer and JEV-R as the forward primer. The viral RNA levels were measured using a one-step real-time reverse transcription (RT)-PCR with SYBR Premix Ex Taq kit (TaKaRa), and the following primers were used $\left(5^{\prime}-3^{\prime}\right)$ : Tag sequence: TTTGCTAGCTTTAGGACCTACTATATCTACCT, JEV-Tag-F primer: TTTGCTAGCTTTAGGACCTACTATATCTACCTG GAATTTGAAGAGGCGCAC JEV-Tag-R primer: TTTGCTAG CTTTAGGACCTACTATATCTACCTGTACTCCACCACGAT GGCTC, JEV-F primer: GGAATTTGAAGAGGCGCAC, JEV-R primer: GTACTCCACCACGATGGCTC, GAPDH-F: AAGGCC ATCACCATCTTCCA, GAPDH-R: GCCAGTAGACTCCACA ACATAC.

\section{In vivo Mouse Experiment}

3-week-old female $\mathrm{BALB} / \mathrm{c}$ mice were obtained from the Vitalriver Company in Beijing, housed in an environmentally controlled room, and maintained on standard laboratory food and water ad libitum throughout the study. To assess the virulence of the JEV mutant, intraperitoneal (i.p.) injections $\left(10^{6} \mathrm{TCID}_{50} / 200 \mu \mathrm{L}\right)$ and intracerebral (i.c.) injections $\left(10^{2}\right.$
$\mathrm{TCID}_{50} / 30 \mu \mathrm{L}$ ) were used. Mouse survival and body weight were monitored daily. Then, three mice were randomly selected from each group after day 7 (i.p.) or 3 (i.c.) post-injection. Mice brain tissues were harvested separately, the virus solution was infected into BHK-21 cells, and brain virus titer was measured using the TCID $_{50}$ method.

\section{Ethics Statement and Biosafety}

All animal experiments involving recombinant JEV were reviewed and approved by the Institutional Animal Care and Use Committee at South China Agricultural University (SCAU) and were carried out in accordance with the approved guidelines.

\section{Statistical Analyses}

Differences between experimental groups were determined using an unpaired $t$-test and analysis of variance (ANOVA) in GraphPad Prism software (GraphPad Software Inc.).

\section{RESULTS}

\section{The Pathogenicity in vivo and Growth Rate in vitro Are Compared Between SA14 and BJB Viruses}

To explore the pathogenicity of $\mathrm{BJB}$ strains isolated from humans, 3-week-old female BALB/c mice were infected with the SA14 and BJB virus strains using $10^{6}$ TCID $_{50}$ by intraperitoneal (i.p.) injection. Mice infected with SA14 virus began to die from 9 days post-infection (dpi), and resulting in a $100 \%$ case fatality rate (Figure 1A). However, mice infected with BJB virus did not die, nor did they display obvious clinical symptoms or weight loss (Figures 1A,B).

In vitro, BHK-21 cells were infected with $\mathrm{SA} 14$ or $\mathrm{BJB}$ at a multiplicity of infection (MOI) of 0.001 . Supernatants and cells were harvested at 24, 48, and 72 hpi. The viral titers were determined by $\mathrm{TCID}_{50}$. As shown in Figure 1C, the growth rate of BJB was higher than SA14 virus.

\section{The Nucleotide Mutations of 3'UTR Significantly Alter the RNA Secondary Structure of SL-IV and DB1 Structure Regions}

The complete genome sequences for the SA14 and BJB virus strains were compared in order to identify nucleotides that consistently differ between these viruses. Through sequence analysis, some mutations at sites 248, 254, 258, and 307 were observed in the $3^{\prime}$ UTR (Table 1). In order to explore the impact of these differences on viral characteristics, we used an infectious cDNA clone pACYC-SA14/U14163 as the parental JEV strain for engineering $3^{\prime}$ UTR (T248C, A254G, A258G, and 307G). The parental virus SA14 (T248, A254, and A258) and recombinant virus rSA14-3'UTRmut (T248C, A254G, A258G and 307G) were rescued, biologically cloned, and sequenced in BHK-21 cells.

Subsequently, we explored whether the mutation of $3^{\prime} \mathrm{UTR}$ would affect the secondary structure of RNA. RNAfold was performed structural prediction of the $3^{\prime} \mathrm{UTR}$ mutant region. According to the prediction of two viral $3^{\prime} \mathrm{UTR}$ secondary 
A

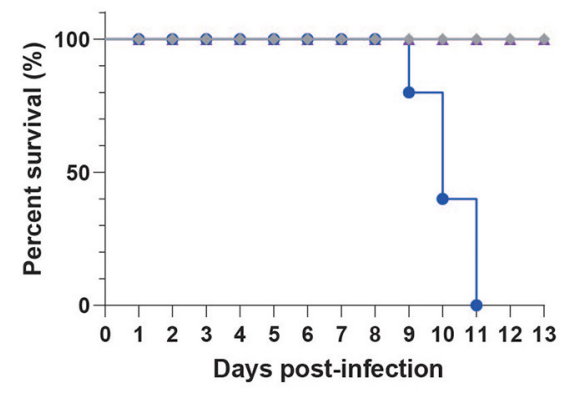

C

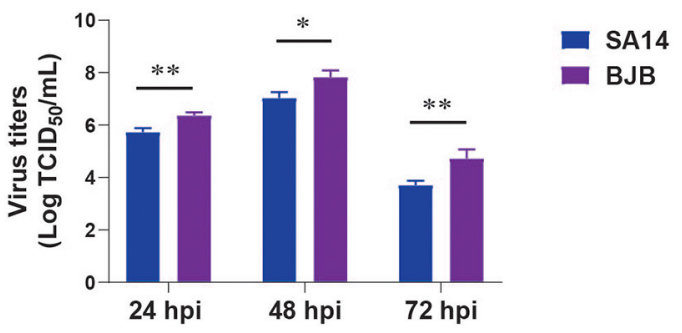

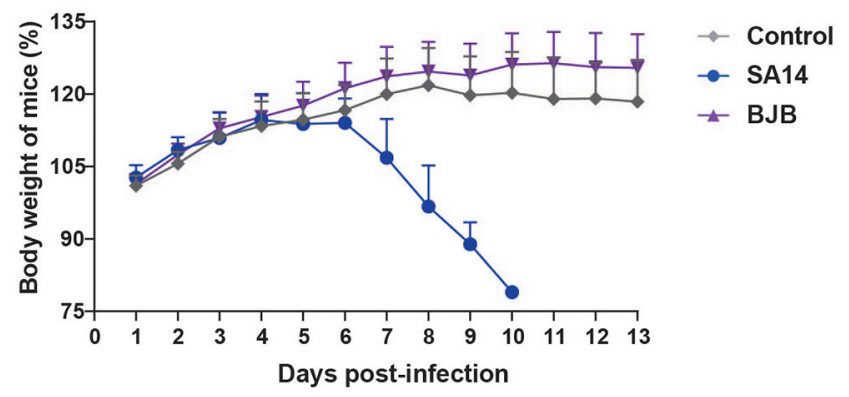

FIGURE 1 | The pathogenicity and growth rate of SA14 and BJB viruses in vivo and in vitro. The SA14 and BJB viruses were injected intraperitoneal into SPF BALB/C $(n=5)$ mice using a dose of $10^{6} \mathrm{TCID}_{50} / 200 \mu \mathrm{L}$. Survival rate $(\mathbf{A})$ and body weight $(\mathbf{B})$ were monitored daily for 13 days. Mice that lost more than $25 \%$ of their initial weight were euthanized. (C) BHK-21 cells seeded in 12-well plates were infected with SA14 or BJB viruses at a multiplicity of infection (MOI) of 0.001. Culture supernatant was then collected at 24,48 , and 72 hpi and subjected to TCID 50 on BHK-21 cells. Data are shown as means \pm SD $(n=3)$, and significance was calculated using an unpaired $t$-test $\left({ }^{\star} P<0.05 ;{ }^{\star \star} P<0.01\right)$.

TABLE 1 | Differences of nucleotide in the 3'UTR between SA14 and BJB.

\begin{tabular}{lcccc}
\hline & \multicolumn{4}{c}{ Nucleotide position $^{\mathbf{a}}$ in the 3'UTR } \\
\cline { 2 - 5 } Virus & $\mathbf{2 4 8}$ & $\mathbf{2 5 4}$ & $\mathbf{2 5 8}$ & $\mathbf{3 0 7}$ \\
\hline SA14 & $\mathrm{T}$ & $\mathrm{A}$ & $\mathrm{A}$ & $-b$ \\
BJB & $\mathrm{C}$ & $\mathrm{G}$ & $\mathrm{G}$ & $\mathrm{G}$ \\
\hline
\end{tabular}

a The nucleotide numbering refers to the start of the $3^{\prime}$ UTR.

$b$ " -" is indicated for a deletion of nucleotides.

structures, the nucleotide sites 248, 254, and 258 were located in the SL-IV structure region, and the nucleotide site 307 was located in the DB1 structure region. The mutations of T248C, $\mathrm{A} 254 \mathrm{G}$, and $\mathrm{A} 258 \mathrm{G}$ reduced the apiculus ring and increased the lateral ring significantly in the SL-IV structure region of $3^{\prime} \mathrm{UTR}$ (Figures 2A,B). Moreover, the insertion of $307 \mathrm{G}$ added a ring to the DB1 structure region. These differences suggested that the nucleotide mutations (T248C, A254G, A258G, and 307G) in 3'UTR significantly alter the RNA secondary structure SL-IV and DB1 regions.

\section{The Nucleotide Mutations of $3^{\prime}$ UTR Promote Viral Growth Rate}

To examine the growth properties of SA14 and rSA14-3'UTRmut in vitro, BHK-21 cells were infected with SA14 or rSA143'UTRmut at a MOI of 0.001. Supernatants and cells were harvested at 24, 48 , and 72 hpi. The viral titers were determined by TCID $_{50}$. As shown in Figure 3A, the titer of rSA14-3'UTRmut virus was higher than the parental virus SA14 by approximately 18 -fold at 72 hpi. Subsequently, replication ability was compared by detecting the level of positive- and negative-chain RNA at 24 hpi, revealing that the positive- (Figure 3B) and negativechain (Figure 3C) RNA levels of rSA14-3'UTRmut were higher than SA14 by approximately 2.5 -fold. These results showed that the nucleotide mutations (T248C, A254G, A258G, and 307G) in 3'UTR promote viral replication.

\section{The Nucleotide Mutations of 3'UTR Reduce the Ability of Neuron Invasion}

In order to investigate changes in the neural invasiveness of the virus in mice, 3-week-old female BALB/c mice were infected with the SA14 and rSA14-3'UTRmut viruses with $10^{6}$ TCID $_{50}$ by i.p., injection. Mice infected with the SA14 virus died beginning at $11 \mathrm{dpi}$, showing signs of illness and weight loss (Figure 4A). Conversely, the rSA14-3'UTRmut virus was attenuated in mice, including only slight weight loss and no case fatality rate. Three mice were randomly selected from each group at $7 \mathrm{dpi}$ to detect the viral burdens in the brain. The titer of SA14 in mice brains reached approximately $10^{6} \mathrm{TCID}_{50}$. However, no virus was detected in mice brains infected with rSA14-3'UTRmut (Figure 4A). These results signified that the nucleotide mutations (T248C, A254G, A258G, and 307G) in $3^{\prime}$ UTR reduce neuron invasion. 

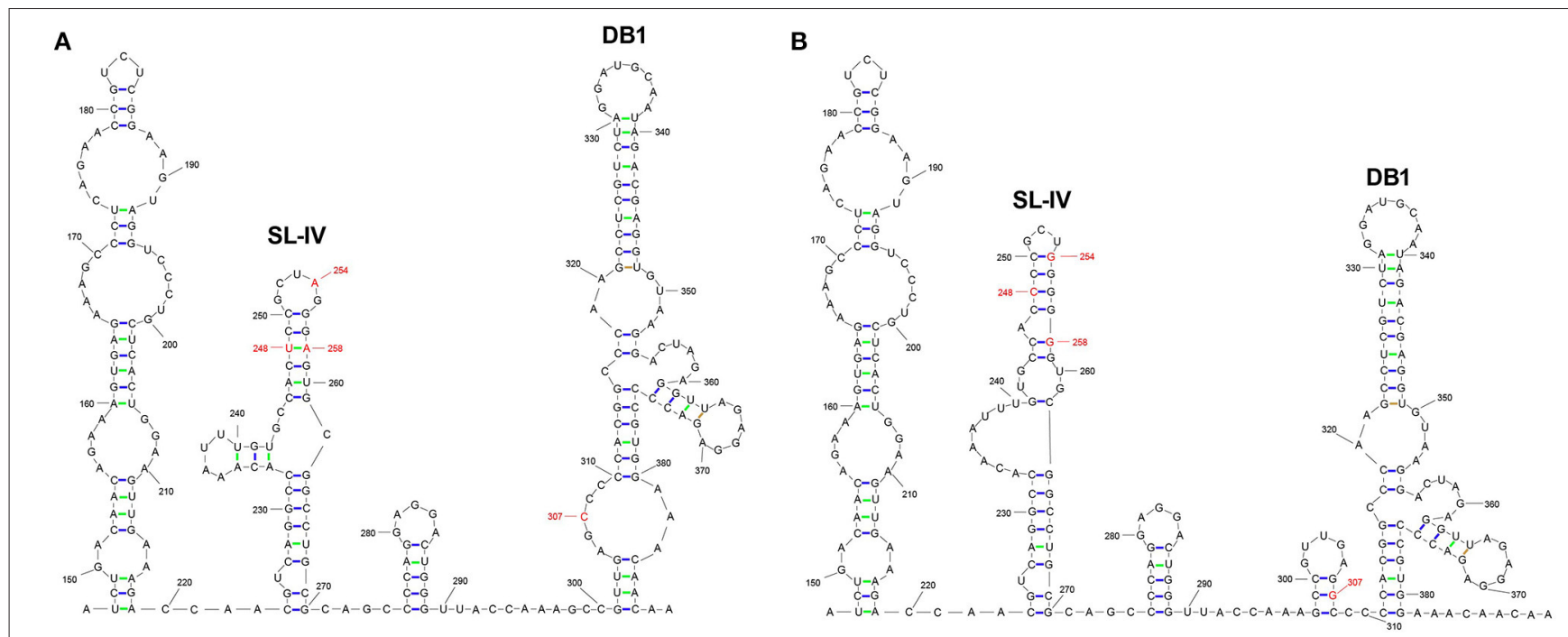

FIGURE 2 | The prediction of RNA secondary structures in 3'UTR SL-IV and DB1 structure regions. The RNA secondary structures with the location of $3^{\prime} U T R$ differences between JEV strains SA14 (A) and rSA14-3'UTRmut (B). RNA structure was used to predict the RNAfold (http://rna.tbi.univie.ac.at//cgi-bin/ RNAWebSuite/RNAfold.cgi) in the mutated regions.
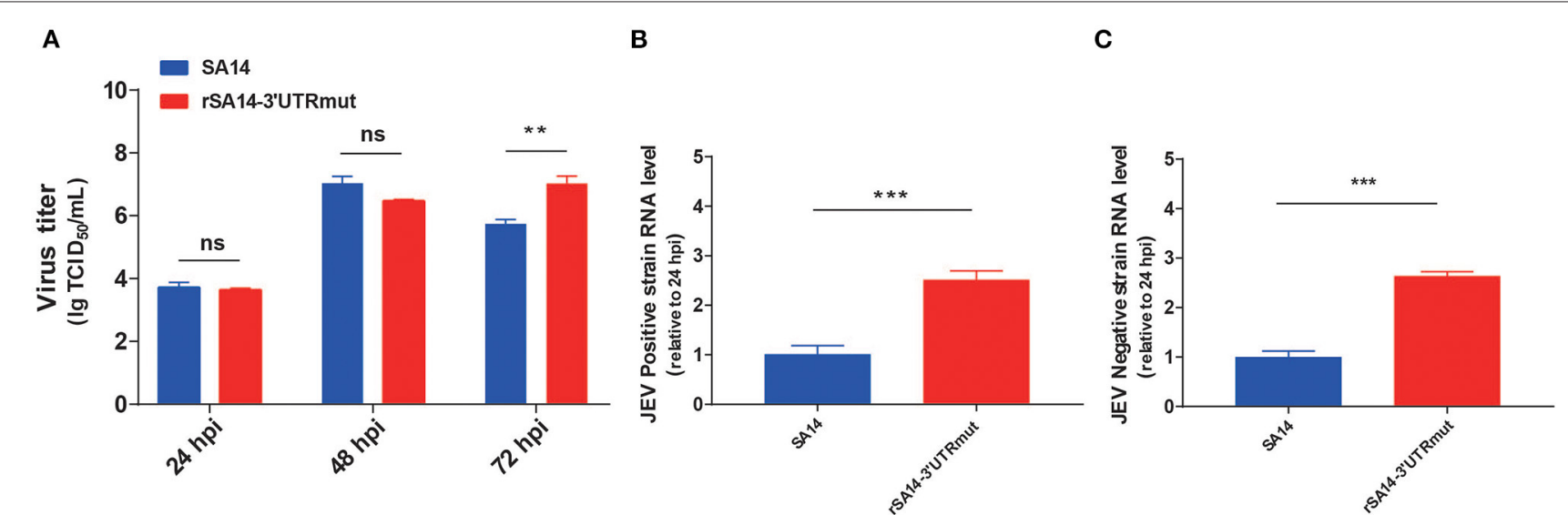

FIGURE 3 | Virus replication of SA14 and rSA14-3'UTRmut. (A) BHK-21 cells seeded in 12-well plates were infected with rSA14-3'UTRmut or parental virus SA14 at a $\mathrm{MOI}$ of 0.001 . Culture supernatant was then collected at 24,48 , and $72 \mathrm{hpi}$ and subjected to $\mathrm{TCID}_{50}$ on $\mathrm{BHK}-21$ cells. BHK-21 cells were infected with viruses at a $\mathrm{MOI}$ of 0.001 , and then cells were harvested to extract total RNA. The obtained RNA was subjected to specific reverse transcription of the positive and negative strands, respectively. The viral positive strands (B) and negative strands (C) RNA levels were measured using a one-step real-time reverse transcription (RT)-PCR. Data are shown as means $\pm \mathrm{SD}(n=3)$, and significance was calculated using an unpaired $t$-test $\left({ }^{* *} P<0.01 ;{ }^{* * * *} P<0.001\right)$.

\section{The Nucleotide Mutations of 3'UTR Decrease Pathogenicity in Mice}

As the above results proved that the nucleotide mutations (T248C, A254G, A258G, and 307G) in 3'UTR are responsible for neural tropism in vivo, we explored the effects of neural pathogenicity in mice. 3-week-old female $\mathrm{BALB} / \mathrm{c}$ mice were infected with the SA14 and rSA14-3'UTRmut viruses with $10^{2}$ $\mathrm{TCID}_{50}$ by intracranially (i.c.) injection. SA14 was virulent in the mice, causing severe weight loss and 100\% mortality (Figure 4B). However, the mortality rate of the rSA14-3'UTRmut virus reached just $20 \%$, with only a slight weight loss. Three mice were randomly selected from each group at $3 \mathrm{dpi}$ to detect the viral burdens in the brain. Strikingly, the rSA14-3'UTRmut virus decreased titers in mice brains by approximately 200 -fold compared with SA14 in mice brains (Figure 4B). These results revealed that the nucleotide mutations (T248C, A254G, A258G, and $307 \mathrm{G}$ ) in $3^{\prime}$ UTR decrease pathogenicity in mice.

\section{DISCUSSION}

In China, before the 1970s and 1980s, the mainstream genotype was G-III. Around 1979, the G-I strains began to be introduced into China and gradually replaced G-III as the mainstream genotype $(6,7)$. Most existing vaccines in the world are G-III, 

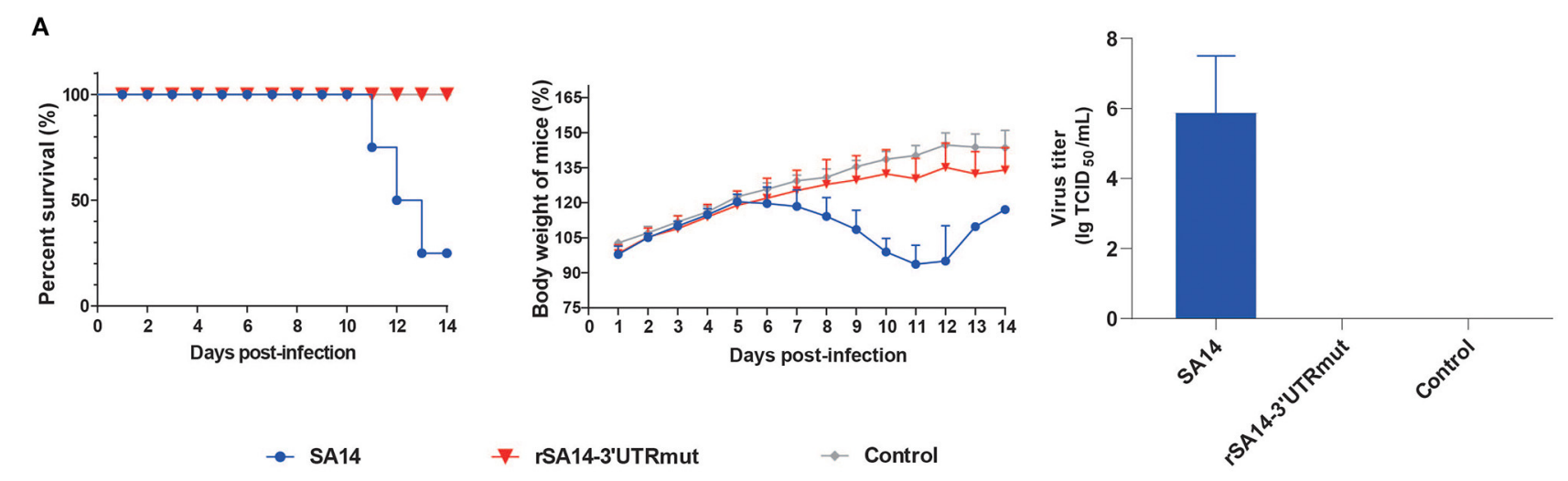

B

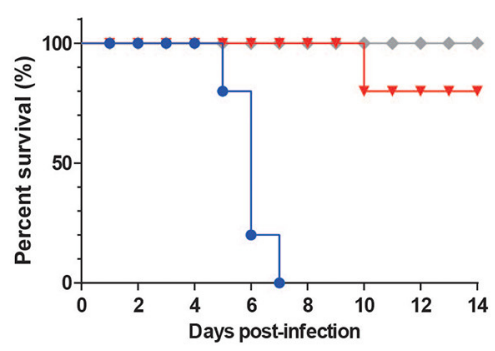

- SA14

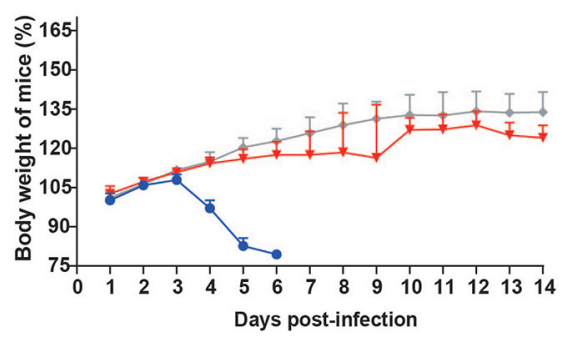

$\leftrightarrow$ Control

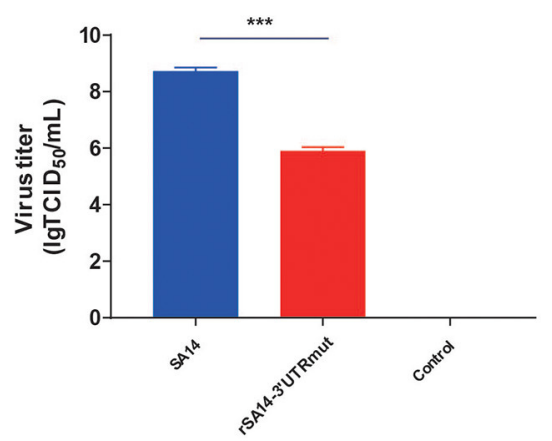

FIGURE 4 | The neuron invasion and pathogenicity in mice. (A) The ability of the virus to invade neurons by the intraperitoneal inoculation route in mice. The SA14 and rSA14-3'UTRmut viruses were injected intraperitoneal into SPF BALB/c mice $(n=8)$ using a dose of $10^{6}$ TCID $50 / 200 \mu L$. Survival rate and body weight were monitored daily for 14 days. Then three mice were randomly selected from each group at day seven post-injection. Mouse brain tissues were harvested separately; the virus solution was infected into BHK-21 cells, and the brain virus titer was measured. (B) The pathogenicity of the virus by the intracranially inoculation route in mice. The SA14 and rSA14-3'UTRmut viruses were injected intracranially into SPF BALB/C mice $(n=8)$ using a dose of $10^{2}$ TCID $50 / 30 \mu L$. Survival rate and body weight were monitored daily for 14 days. Then three mice were randomly selected from each group at day 3 post-injection. Mouse brain tissues were harvested separately; the virus solution was infected into BHK-21 cells, and the brain virus titer was measured. Mice that lost more than $25 \%$ of their initial weight were euthanized. The percentage weight from each group and each time point are presented as means $\pm \operatorname{SD}(n=3)$ and the significance was calculated using an unpaired $t$-test $\left({ }^{\star \star \star} P<0.001\right)$.

which can completely protect against the G-III strain. However, studies have shown that the current G-III vaccine does not provide complete protection against the G-I strain, and the GI vaccine also does not provide complete protection against the G-III strain $(8,9)$. Hence, the development of a new vaccine is particularly important. However, the requirements for the new vaccine include low virulence, high replication and strong immunogenicity. In this study, we compared pathogenicity between Japanese encephalitis virus SA14 and BJB (isolated from humans in the 1970s) strains, and we found that the BJB strain was attenuated in mice, with no case fatality rate. Strinkingly, the growth rate of BJB was higher than SA14.

Based on the sequence alignment of the viral genome between SA14 and BJB virus strains, some mutations at sites 248, 254, 258, as well as an insert at site 307 were observed in $3^{\prime}$ UTR.
The untranslated regions of flavivirus play a significant role in the virus life cycle. The study of RNA elements formed in the untranslated regions of other members of the Flaviviridae family (DENV, WNV, etc.) revealed the important functions in virus replication and significant impacts on lesion and mice pathogenicity $(17,29-31)$.

Subsequently, we predicte RNA secondary structure using RNAfold. The analysis showed that the nucleotide sites 248 , 254, and 258 are located in the SL-IV structure region, and the nucleotide mutation 307 is located in the DB1 structure region. The nucleotide mutations T248C, A254G, and A258G cause the top loop of the SL-IV structure to shrink and the side loop to increase. The insertion of $307 \mathrm{G}$ adds a ring to the DB1 structure.

We became curious as to whether this secondary structure change affects the biological properties of the virus. We used an 
infectious cDNA clone (pACYC-SA14/U14163) as the parental JEV strain for engineering 3'UTR (T248C, A254G, A258G and $307 \mathrm{G}$ ), and we rescued the mutant virus, which was named rSA14-3'UTRmut. The growth rate of the rSA14-3'UTRmut virus was higher than the parental virus SA14 at $72 \mathrm{hpi}$. However, there was no significant difference between 24 and 48 hpi. We speculated that the mutant virus would cause less cell lesions in the later growth period, which was conducive to the replication of the virus. The positive- and negative-chain RNA levels of rSA143'UTRmut were significantly higher than SA14 at $24 \mathrm{hpi}$. These results showed that the nucleotide mutations (T248C, A254G, A258G and 307G) in 3'UTR promote viral replication.

Subsequently, we investigated the changes in the neural invasiveness of the virus in mice. We found that the rSA14$3^{\prime}$ UTRmut virus was attenuated in mice, including only slight weight loss and no case fatality rate, this compared to SA14, which caused death, illness and weight loss. The mice infected with SA14 started to die at 11 dpi not at 6 dpi as Figure 1A. We speculated that it might be due to individual differences, as well as differences in ambient temperature and humidity. Further, no virus was detected in mice brains infected with rSA14$3^{\prime}$ UTRmut at $7 \mathrm{dpi}$. This indicated that the nucleotide mutations (T248C, A254G, A258G, and 307G) in 3'UTR reduce the ability of neuron invasion. We then explored the effects of the neural pathogenicity in mice. SA14 was virulent in mice, including severe weight loss and $100 \%$ mortality. However, the mortality rate of the rSA14-3'UTRmut virus reached just $20 \%$ with only slight weight loss. The rSA14-3' UTRmut virus decreased viral burdens in mice brains by approximately 200 -fold compared with SA14. The neuron tropism of the rSA14-3' UTRmut virus in neuron cells in vitro needs further study. The reduction in mouse pathogenicity caused by changes in secondary structure may be due to SL-IV preventing Xrn1 from reducing the efficiency of viral RNA degradation and reducing sfRNA production, making the virus more susceptible to natural immunity and clearance $(19,32)$. The specific mechanism still needs further exploration and verification.

In summary, our study found that the SL-IV and DB1 regions of $3^{\prime}$ UTR are essential for JEV replication, neural invasiveness,

\section{REFERENCES}

1. Weaver SC, Reisen WK. Present and future arboviral threats. Antivir Res. (2010) 85:328-45. doi: 10.1016/j.antiviral.2009.10.008

2. van den Hurk AF, Ritchie SA, Mackenzie JS. Ecology and geographical expansion of Japanese encephalitis virus. Annu Rev Entomol. (2009) 54:1735. doi: 10.1146/annurev.ento.54.110807.090510

3. Vaughn DW, Hoke CH. The epidemiology of Japanese encephalitis: prospects for prevention. Epidemiol Rev. (1992) 14:197-221. doi: 10.1093/oxfordjournals.epirev.a036087

4. Tsarev SA, Sanders ML, Vaughn DW, Innis BL. Phylogenetic analysis suggests only one serotype of Japanese encephalitis virus. Vaccine. (2000) 18(Suppl. 2):36-43. doi: 10.1016/S0264-410X(00)00039-6

5. Uchil PD, Satchidanandam V. Phylogenetic analysis of Japanese encephalitis virus: envelope gene based analysis reveals a fifth genotype, geographic clustering, and multiple introductions of the virus into the Indian subcontinent. Am J Trop Med Hyg. (2001) 65:242-51. doi: 10.4269/ajtmh.2001.65.242 and viral pathogenicity. Our results confirm that the nucleotide mutations (T248C, A254G, A258G, and 307G) in 3'UTR can promote viral replication in BHK-21 cells, reduce the ability of neuron invasion, and decrease pathogenicity in mice. This study provides a new perspective for weakening candidate vaccine strains and increasing virus production.

\section{DATA AVAILABILITY STATEMENT}

The original contributions generated for this study are included in the article/supplementary material, further inquiries can be directed to the corresponding author/s.

\section{ETHICS STATEMENT}

All animal experiments involving recombinant JEV were reviewed and approved by the Institutional Animal Care and Use Committee at South China Agricultural University (SCAU) and were carried out in accordance with the approved guidelines.

\section{AUTHOR CONTRIBUTIONS}

JX, YZ, WQ, and ML conceived and designed the experiments and wrote the manuscript. JX, YZ, ZL, LL, QX, and JL performed the experiments. JX, YZ, ZY, CH, and WQ analyzed the results of the experiments. All authors read and approved the final manuscript.

\section{FUNDING}

This work was partially supported by The National Key Research and Development Program of China (2016YFD0500405), the National Natural Science Foundation (NSFC) of China (31272563), the Key Laboratory of Fujian Animal Diseases Control (2017), Guangdong Province Universities and Colleges Pearl River Scholar Funded Scheme (2018), and Young Scholars of Yangtze River Scholar Professor Program (2019, WQ).
6. Han N, Adams J, Chen P, Guo Z, Zhong X, Fang W, et al. Comparison of genotypes I and III in Japanese encephalitis virus reveals distinct differences in their genetic and host diversity. J Virol. (2014) 88:1146979. doi: 10.1128/JVI.02050-14

7. Schuh AJ, Ward MJ, Leigh Brown AJ, Barrett ADT. Dynamics of the emergence and establishment of a newly dominant genotype of Japanese encephalitis virus throughout Asia. J Virol. (2014) 88:452232. doi: 10.1128/JVI.02686-13

8. Fan Y, Chen J, Chen Y, Lin J, Chiou S. Reduced neutralizing antibody titer against genotype I virus in swine immunized with a live-attenuated genotype III Japanese encephalitis virus vaccine. Vet Microbiol. (2013) 163:248-56. doi: 10.1016/j.vetmic.2013.01.017

9. Wei J, Wang X, Zhang J, Guo S, Pang L, Shi K, et al. Partial crossprotection between Japanese encephalitis virus genotype I and III in mice. PLoS Neglect Trop. D. (2019) 13:e7601. doi: 10.1371/journal.pntd. 0007601

10. Sampath A, Padmanabhan R. Molecular targets for flavivirus drug discovery. Antivir Res. (2009) 81:6-15. doi: 10.1016/j.antiviral.2008.08.004 
11. Wang X, Li S, Zhu L, Nian Q, Yuan S, Gao Q, et al. Near-atomic structure of Japanese encephalitis virus reveals critical determinants of virulence and stability. Nat Commun. (2017) 8:14. doi: 10.1038/s41467-017-00024-6

12. Avirutnan P, Punyadee N, Noisakran S, Komoltri C, Thiemmeca S, Auethavornanan $\mathrm{K}$, et al. Vascular leakage in severe dengue virus infections: a potential role for the nonstructural viral protein NS1 complement. [Comparative Study; Journal Article; Research Support, Non-U.S. Gov't]. J Infect Dis. (2006) 193:1078-88. doi: 10.1086/500949

13. Ye J, Zhu B, Fu ZF, Chen H, Cao S. Immune evasion strategies of flaviviruses. Vaccine. (2013) 31:461-71. doi: 10.1016/j.vaccine.2012.11.015

14. Ye Q, Li XF, Zhao H, Li SH, Deng YQ, Cao RY, et al. A single nucleotide mutation in NS2A of Japanese encephalitis-live vaccine virus (SA14-142) ablates NS1' formation contributes to attenuation. J Gen Virol. (2012) 93:1959-64. doi: 10.1099/vir.0.043844-0

15. Shi PY, Brinton MA, Veal JM, Zhong YY, Wilson WD. Evidence for the existence of a pseudoknot structure at the 3' terminus of the flavivirus genomic RNA. [Journal Article; Research Support, Non-U.S. Gov't; Research Support, Gov't US. P.H.S.]. Biochemistry. (1996) 35:4222-30. doi: 10.1021/bi952398v

16. Chang R, Hsu T, Chen Y, Liu S, Tsai Y, Lin Y, et al. Japanese encephalitis virus non-coding RNA inhibits activation of interferon by blocking nuclear translocation of interferon regulatory factor 3. Vet Microbiol. (2013) 166:1121. doi: 10.1016/j.vetmic.2013.04.026

17. Roby JA, Pijlman GP, Wilusz J, Khromykh AA. Noncoding subgenomic flavivirus RNA: multiple functions in West Nile virus pathogenesis and modulation of host responses. Viruses. (2014) 6:404-27. doi: 10.3390/v6020404

18. Uzri D, Gehrke L. Nucleotide sequences and modifications that determine RIG-I/RNA binding and signaling activities. J Virol. (2009) 83:417484. doi: 10.1128/JVI.02449-08

19. Clarke BD, Roby JA, Slonchak A, Khromykh AA. Functional non-coding RNAs derived from the flavivirus $3^{\prime}$ untranslated region. Virus Res. (2015) 206:53-61. doi: 10.1016/j.virusres.2015.01.026

20. Alvarez DE, De Lella Ezcurra AL, Fucito S, Gamarnik AV. Role of RNA structures present at the $3^{\prime} \mathrm{UTR}$ of dengue virus on translation, RNA synthesis, viral replication. Virology. (2005) 339:200-12. doi: 10.1016/j.virol.2005.06.009

21. Göertz GP, Fros JJ, Miesen P, Vogels CBF, van der Bent ML, Geertsema C, et al. Noncoding subgenomic flavivirus RNA is processed by the mosquito RNA interference machinery determines west nile virus transmission by culex pipiens mosquitoes. J Virol. (2016) 90:10145-59. doi: 10.1128/JVI.00930-16

22. Tajima S, Nukui Y, Takasaki T, Kurane I. Characterization of the variable region in the $3^{\prime}$ non-translated region of dengue type 1 virus. J Gen Virol. (2007) 88:2214-22. doi: 10.1099/vir.0.82661-0

23. Tumban E, Mitzel DN, Maes NE, Hanson CT, Whitehead SS, Hanley KA. Replacement of the 3' untranslated variable region of mosquito-borne dengue virus with that of tick-borne Langat virus does not alter vector specificity. $J$ Gen Virol. (2011) 92:841-8. doi: 10.1099/vir.0.026997-0

24. Yun S, Choi Y, Song B, Lee Y. $3^{\prime}$ cis-Acting elements that contribute to the competence efficiency of japanese encephalitis virus genome replication: functional importance of sequence duplications, deletions, substitutions. $J$ Virol. (2009) 83:7909-30. doi: 10.1128/JVI.02541-08
25. Cumberworth SL, Clark JJ, Kohl A, Donald CL. Inhibition of type I interferon induction and signalling by mosquito-borne flaviviruses. [Journal Article; Review; Research Support, Non-U.S. Gov't]. Cell Microbiol. (2017) 19:e12737. doi: 10.1111/cmi.12737

26. Moon SL, Anderson JR, Kumagai Y, Wilusz CJ, Akira S, Khromykh AA, et al. A noncoding RNA produced by arthropod-borne flaviviruses inhibits the cellular exoribonuclease XRN1 and alters host mRNA stability. RNA. (2012) 18:2029-40. doi: 10.1261/rna.034330.112

27. Schuessler A, Funk A, Lazear HM, Cooper DA, Torres S, Daffis S, et al. West nile virus noncoding subgenomic RNA contributes to viral evasion of the type i Interferon-Mediated antiviral response. J Virol. (2012) 86:570818. doi: 10.1128/JVI.00207-12

28. Men R, Bray M, Clark D, Chanock RM, Lai CJ. Dengue type 4 virus mutants containing deletions in the $3^{\prime}$ noncoding region of the RNA genome: analysis of growth restriction in cell culture and altered viremia pattern and immunogenicity in rhesus monkeys. J Virol. (1996) 70:39307. doi: 10.1128/jvi.70.6.3930-3937.1996

29. de Borba L, Villordo SM, Marsico FL, Carballeda JM, Filomatori CV, Gebhard LG. et al. RNA structure duplication in the dengue virus $3^{\prime}$ UTR: redundancy or host specificity? mBio. (2019) 10:e2506-18. doi: 10.1128/mBio. 02506-18

30. Ng WC, Soto-Acosta R, Bradrick SS, Garcia-Blanco MA, Ooi EE. The 5' and 3' untranslated regions of the flaviviral genome. Viruses. (2017) 9:137. doi: 10.3390/v9060137

31. Villordo SM, Carballeda JM, Filomatori CV, Gamarnik AV. RNA structure duplications and flavivirus host adaptation. Trends Microbiol. (2016) 24:270 83. doi: 10.1016/j.tim.2016.01.002

32. Funk A, Truong K, Nagasaki T, Torres S, Floden N, Balmori Melian $\mathrm{E}$, et al. RNA structures required for production of subgenomic flavivirus RNA. J Virol. (2010) 84:11407-17. doi: 10.1128/JVI.01 $159-10$

Conflict of Interest: The authors declare that the research was conducted in the absence of any commercial or financial relationships that could be construed as a potential conflict of interest.

Publisher's Note: All claims expressed in this article are solely those of the authors and do not necessarily represent those of their affiliated organizations, or those of the publisher, the editors and the reviewers. Any product that may be evaluated in this article, or claim that may be made by its manufacturer, is not guaranteed or endorsed by the publisher.

Copyright (C) 2021 Xing, Zhang, Lin, Liu, Xu, Liang, Yuan, Huang, Liao and Qi. This is an open-access article distributed under the terms of the Creative Commons Attribution License (CC BY). The use, distribution or reproduction in other forums is permitted, provided the original author(s) and the copyright owner(s) are credited and that the original publication in this journal is cited, in accordance with accepted academic practice. No use, distribution or reproduction is permitted which does not comply with these terms. 